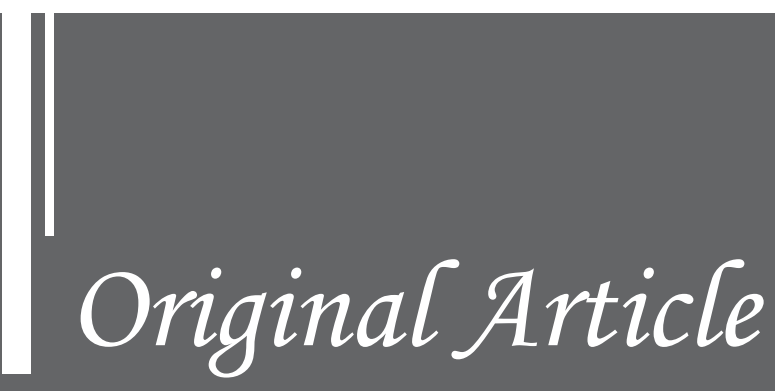

\title{
AN EPIDEMIOLOGICALANALYSIS OF LABORATORY CONFIRMED CASES OF CHIKUNGUNYA, PAKISTAN, 2016-2018
}

\author{
Nosheen Ashraf', Sana Habib Abbasi ${ }^{1}$, Fariha Munir ${ }^{3}$, Amna Ali1, M. \\ Akram Qamar', Naveed Syed ${ }^{2}$, Jamil A. Ansari', Mumtaz Ali khan', \\ Rana M.Safdar ${ }^{1}$, Aamer Ikram ${ }^{1}$
}

${ }^{1}$ National Institute of Health, Islamabad, Pakistan

${ }^{2}$ Public Health England

${ }^{3}$ Pakistan Biological Safety Association, Pakistan

Corresponding Author: Nosheen Ashraf, Email: nosheenawan@hotmail.com

\section{Abstract}

Background: Chikungunya is a vector-borne disease that is spread by the mosquito Aedes aegypti and Aedes albopictus. Disease mostly occurs in Africa, Asia and the Indian subcontinent. Recent outbreak of chikungunya was reported in 2017 in Karachi, Pakistan, after which sporadic cases were reported from different areas of Pakistan. There are very limited demographic data available for chikungunya in Pakistan and therefore, further information is required. The current study will provide latest information to assist public health professionals and policy makers in order to provide effective management of cases by means of appropriate resource.

Methods: In this cross sectional study, information regarding 750 laboratory confirmed cases from Dec 2016 to Apr 2018 were collected from the virology laboratory, NIH. Data regarding age, sex, reporting facility, district and province were collected. Data entry and cleaning was done in Microsoft excel and descriptive analysis using epiInfo. The data were grouped, tabulated and represented graphically while geographical location of the cases was mapped.

Results: A total of 403(53.73\%) cases were male and 347 (46.27\%) female with an age range from 0.6 to 80 years and the mean age was $(32.5+-17.5)$. The most affected age group was 20 to 29 Years. Sindh remained the most affected province with $494(65.87 \%)$ cases reported followed by KP with $167(22.27 \%)$ cases. The highest number of cases [148(19.7\%)] were reported in May 2017.

Conclusion: Provision and sharing of accurate and timely data can reduce the risk and spread of the disease.

Keywords: chikungunya, age group, temperature, Pakistan 


\section{Introduction}

Chikungunya is a vector-borne disease that is spread by the mosquito Aedes aegypti and Aedes albopictus. Chikungunya fever is induced by the chikungunya virus, which is an alpha virus from the Togaviridae family (1). Extensive global movement and trade have harnessed the potential of A. albopictus to move to new geographical areas and to successfully establish endemicity (2). Although many mosquitoes are involved in the transmission of chikungunya virus however A. aegypti and A. albopictus are chiefly associated with the outbreaks of chikungunya worldwide. A. aegypti was the primary vector involved in the mosquito associated outbreaks in India during 2005-2006. In the tropical regions A. aegypti is prevalent while A. albopictus are also found in temperate regions. Due to the potential of rapid adaptation of chikungunya virus, it might be better adapted for A albopictus which would increase the possibility of global spread of chikungunya (3). Previously it was thought that these mosquito species only breed on clean waters however, recently the vectors have adapted to thrive on polluted water bodies as well (4).

The virus is recognized to give rise to sporadic and sudden outbreaks but unfortunately there are no licensed treatments or vaccines for this disease (4-6). The chikungunya infection is characterized by sudden onset of fever followed by severe polyarthralgia (6). Arthralgia is a characteristic clinical feature of chikungunya (7) whereas less frequent complications include gastrointestinal, cardiac and neurological problems (8). Very rare vertical transmission of infection from mother to child has been observed (1). Secondly chikungunya is often misdiagnosed or confused with dengue as both the diseases share similar vectors, clinical manifestations, geographical areas and seasonal patterns $(2,16)$. Similarities in dengue and chikungunya make the clinical diagnosis more problematic but the morbidity and joint involvement is more pronounced and prolonged in chikungunya. Chikungunya has increased dramatically over recent years in incidence and geographical coverage (7). Pakistan is rife for many vector borne diseases due to factors such as favorable climate, poor sanitation and improper garbage disposal - which all stimulate vector proliferation. (9). The first recorded Chikungunya virus detection in Pakistan was in 1983 when antibodies against the virus were identified in rodents and humans (10). However, in November 2016, Pakistan experienced an epidemic of chikungunya in the megacity of Karachi affecting approximately 30,000 individuals with 4000 locally confirmed cases by RT PCR (4). However, three cases were verified by National Institute of Health (NIH) as the first cases of disease in Pakistan and later reported 82 confirmed and 818 suspected cases by Feb 2017 $(4,6,16)$. According to the weekly report generated by Field Epidemiology and Lab Training Program (FELTP) Pakistan, a total of 8521 cases have been recorded throughout the country from December 2016- April 2018. Our study includes the laboratory confirmed cases of chikungunya from Pakistan with the samples checked and confirmed by NIH, Islamabad, Pakistan.

The warm winters of Karachi $\left(21.5-22.5^{\circ} \mathrm{C}\right)$ and poor sanitary conditions favor the proliferation and propagation of chikungunya virus (4) (11). The incidence of chikungunya virus varies widely by age and sex (12), as well as geographical locations (Pakistan's varied environmental and climatic conditions) (16). Following the 2016 chikungunya outbreaks in Pakistan no subsequent confirmed outbreaks have been recorded to date. An international team visited Karachi which highlighted the poor cleanliness and sanitary conditions of the city significantly contributing to the spread of this new disease. The aim of this study is to provide updated disease information and to review the epidemiology for chikungunya in Pakistan.

Methodology

Study Design And Study Population

A cross sectional study was conducted from the year 2016-2018 using laboratory confirmed positive cases of chikungunya reported by the Public Health laboratory, National Institute of Health (NIH) Islamabad from Dec 2016- Apr 2018 inclusive. The study includes reported cases from 17 districts of Pakistan, covering both the northern and southern parts of the country. Case, age and notification month was identified and stratified geographically to document the burden of disease in each part of the country.

Case Definition

Suspected chikungunya case was defined as a person having acute onset of fever $>38.5^{\circ} \mathrm{C}$ within 7 days of the mosquito bite, with rash and severe arthralgia/arthritis reported from different hospitals and outdoor clinics across Pakistan, between November 2016- April 2018 tested in NIH laboratory, Islamabad.

Lab Confirmation

Viral RNA was extracted using QI Amp viral RNA minikit (Qiagen, $\mathrm{GmbH}$ ) from sera following manufacturers protocol and tested by onestep real-time reverse transcription-polymerase chain reaction (rRTPCR) on Applied Biosystems platform (ABI7500) following US-CDC Trioplex rea time RT-PCR (Dengue, Chikungunya and Zika) assay at NIH, Pakistan.

Data analysis.

The data were entered in Microsoft Excel ${ }^{\circledR}$ and analysis was done using Epi Info® version7.

Results

Of 1437 suspected chikungunya fever (CHKF) cases nationwide 750 $(52.2 \%)$ were confirmed CHKF patients. Out of these 1437 suspected cases $830(57.8 \%)$ were males and $607(42.2 \%)$ were females. Figure-1 shows the gender distribution of the CHKF suspected and confirmed cases with males being slightly more affected.

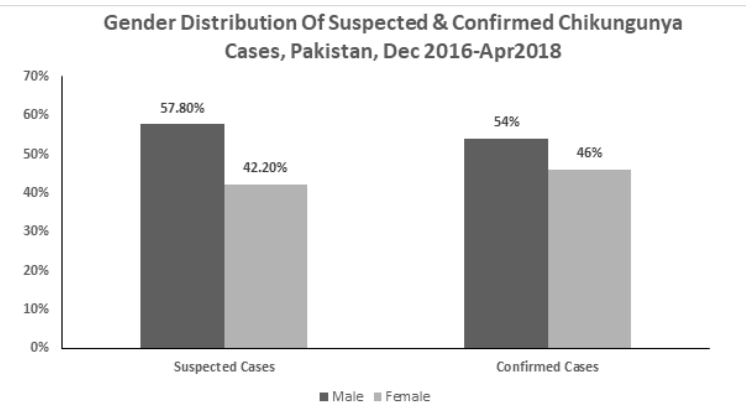

Figure 1: Gender distribution of Suspected \& Confirmed Chikungunya Cases in Pakistan from Dec 2016-Apr 2018

Table 1 gives the province wise distribution of CHKF cases in Pakistan. Maximum number of cases came from Sindh as there was an outbreak of CHKF during that period.

Table 1: Province wise distribution of confirmed Chikungunya cases in Pakistan from Dec 2016-Apr 2018

\begin{tabular}{|l|c|c|}
\hline Province/Area & Number of Cases & Percentages \\
\hline Baluchistan & 30 & $1.20 \%$ \\
\hline Islamabad & 26 & $3.47 \%$ \\
\hline KPK & 167 & $22.27 \%$ \\
\hline Punjab & 54 & $7.20 \%$ \\
\hline Sindh & 473 & $65.87 \%$ \\
\hline Total & $\mathbf{7 5 0}$ & $100.00 \%$ \\
\hline
\end{tabular}

Figure 2. Shows the district distribution of CHKF cases in Pakistan, with the epidemic in Karachi contributing to $41.3 \%$ of total cases in this period followed by $21.07 \%$ of the total cases in Tharparkar.

District/Area Wise Distribution Of Chikungunya Cases, Pakistan, Dec 2016-Apr 2018

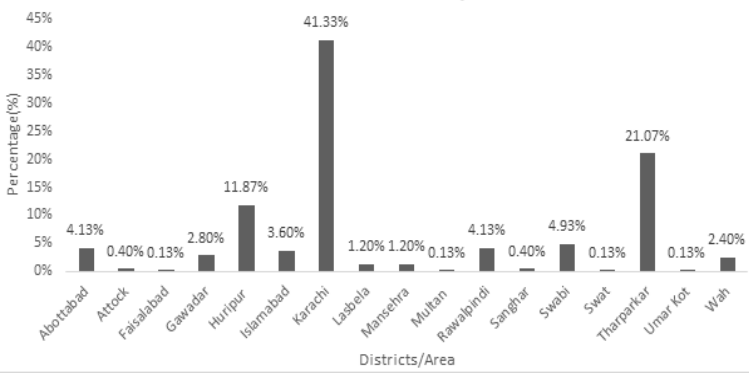

Figure 2: District distribution of confirmed Chikungunya cases in Pakistan from Dec 2016-Apr 2018

Figure 3. Shows the temporal trend of CHKF cases. Maximum number of cases were observed in temperate conditions i.e. in May, August and September 148(19.7\%), 112(14.9\%), 119(15.9\%) cases respectively. 


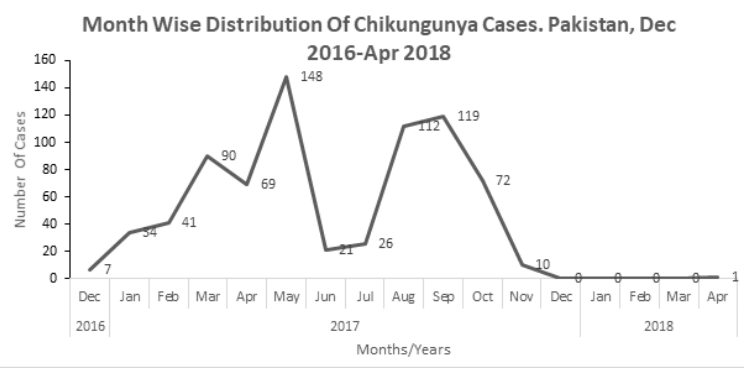

Figure 3: Temporal distribution of confirmed Chikungunya cases in Pakistan from Dec 2016-Apr 2018

Figure 4 shows the age and gender distribution of confirmed CHKF cases with $529(71.87 \%)$ cases aged between 10 to 49 years and being most common in 20 to 29 year olds with $163(21.73 \%)$ cases.

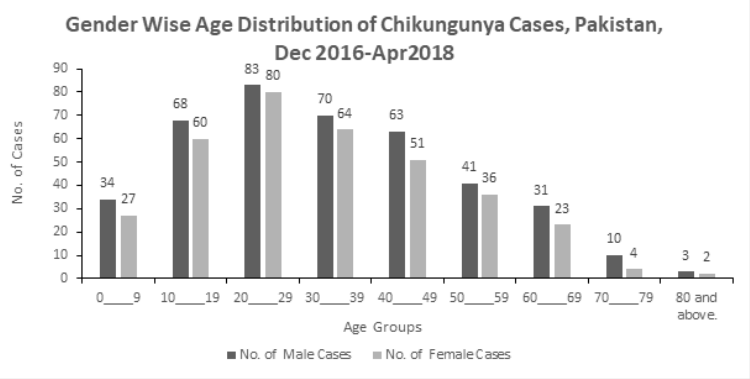

Figure 4: Gender and age distribution of Chikungunya cases in Pakistan from Dec 2016-Apr 2018

Figure 5 shows the duration of illness associated with confirmed CHKF cases. About $73.7 \%$ of the affected patients suffered from 6 to 8 weeks.

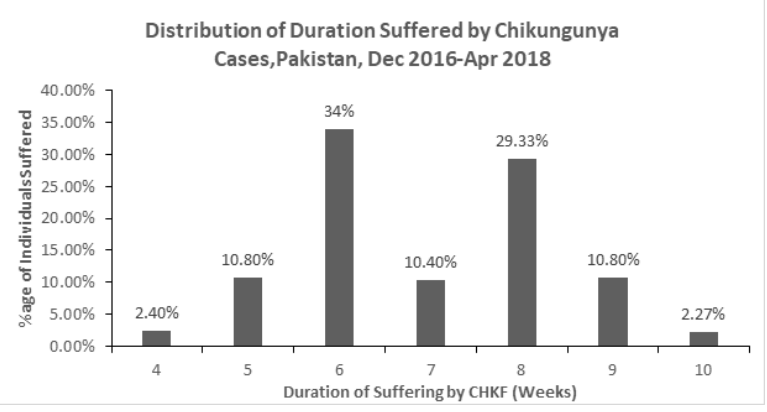

Figure 5: Duration of symptoms of Chikungunya

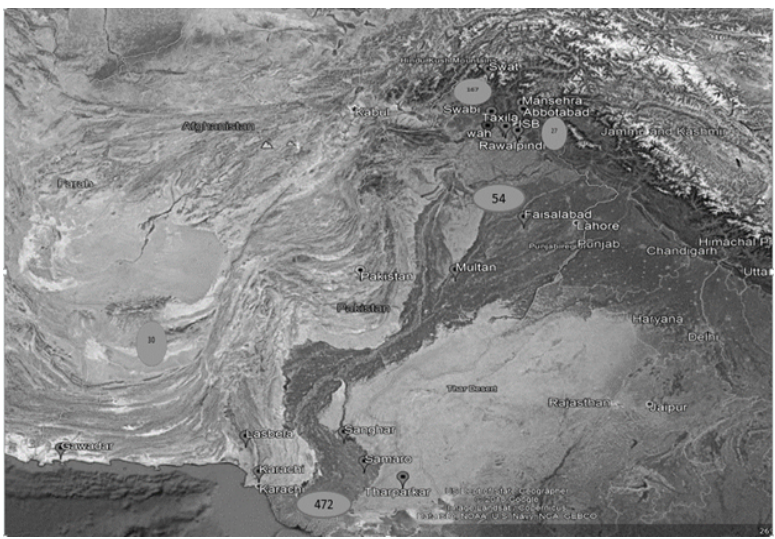

Figure 6: Geographical distribution of Chikungunya cases in Pakistan from Dec 2016-Apr 2018
Gender and CHKF were tested for the possible association and a marginal association was found between the two attributes $(\mathrm{X} 2=10.422, \mathrm{df}=1, \mathrm{P}$-value $=0.001, \mathrm{Phi}=-0.085)$ where $347(57.2 \%)$ of the females out of 607 females suspected for CHKF and 403(48.6\%) of the male were CHKF patients out of 830 males suspected for CHKF. The relative risk for getting CHKF for females was 1.177 times higher compare to male with $95 \%$ C.I $(1.067,1.299)$.

Mean fever duration in CHKF patients was 4.96 days with $95 \%$ C.I $(4.89,5.04)$ days. In average duration of fever for male and female were 5.05 days and 4.87 days respectively. There was significant difference observed in duration of fever for male and female $(\mathrm{t}=2.415, \mathrm{df}=748, \mathrm{P}$ value $=0.016)$ with $95 \%$ C.I $(0.0339,0.3288)$ days

In average a patient suffered for 48.8 days with $95 \%$ C.I $(48.1,49.5)$ days with no significant difference between the sexes

Discussion

The chikungunya virus has an RNA positive stranded genome and hence it is replicated by the help of RNA dependent RNA polymerase, as there is no proof-reading mechanism the virus genome modifies itself and adapts rapidly and efficiently to the changing conditions. The lack of proofreading by the RNA polymerase and its ability to mutate and adapt, chikungunya virus has become a major threat worldwide (3). Climatic conditions play a significant role in the spread and proliferation of the virus, it was noted that the areas which have a warm and humid climate like Sindh reported to have most of the cases $(65 \%$ cases). Similarly Karachi experienced outbreak of CHKF due to its favorable climatic conditions for the vector and virus. Regarding the sporadic cases of chikungunya across the country had the travel history from epidemic areas mainly from Karachi. According to a report, the regions of South Asia experience unbounded epidemics due to the humid and warm climatic conditions, poor municipal management and inappropriate water storage, stagnant water at different places (13). The elevated incidence of disease by the mosquito Aedes albopictus in South Asia and improved endurance of Aedes aegypti is due to the excessive use of plastic bags and tires for water storage, this malpractice has exposed the population to numerous vector borne diseases (14).

The analysis of demographic profile of this study revealed that out of 750 chikungunya positive cases, more males were affected as compared to females $(54 \%)$ and the age group that suffered the most from the chikungunya infection was 20-30 years. These findings were similar to a prospective study conducted in North India, according to the findings similar age group was most affected and reported a higher frequency of males who experienced the disease (15). The males are more socially active in the South Asian regions and hence spend more time outside the house hence the increased outdoor exposure results in more males acquiring the vector or mosquito borne diseases.

According to the results of present study a peak of chikungunya cases was observed during the month of May 2017 and later in Aug - Sept 2017. On an average Karachi, a metropolitan city of Pakistan experience $74 \%$ of humidity and $29 \mathrm{oC}$ temperature during these months, which are ideal for mosquito to breed. This time period is also the time of summer vacations and traveling across the country is more common. Conversely et al reported that the high incidence of chikungunya was noted in the month of November, when India has a pre-monsoon or monsoon season prevailing (15). Another reason for outbreaks and high incidence of infectious diseases in metropolitan cities is that many people travel from sub urban and rural areas to seek medical care. Lack of infrastructure and facilities in these areas harness the spread of diseases.

\section{Conclusion and recommendation}

Based on the results of this research paper, it can be concluded that the peak season for chikungunya infection is the monsoon season (AugustSeptember) in Pakistan when both the temperature as well as humidity levels favor the spread of the disease. From the results of this research, the most vulnerable age group was identified to be in their $20 \mathrm{~s}$, although more research is required on this particular variable. The investigation department of Pakistan should launch timely and effective surveillance for chikungunya. Provision of accurate and timely data can reduce the risk and spread of the disease. Launching of awareness campaigns to increase the knowledge of people regarding prevention and training of professionals for better case diagnosis and management.

Limitations

These cases are not representative of the whole population as these were reported from only a few areas of Pakistan due to absence of 
proper surveillance across the country. Also only cases reported to Virology lab NIH were studied which further compromise representativeness of the results.

No funding was required for this research paper.

Acknowledgments: No acknowledgments required.

References

1. Sahibzada HA, Khurshid Z, Khan RS, Sohail M, Siddiqi KM. Short Communication Outbreak Of Chikungunya Virus In Karachi, Pakistan. 2018;30(3):486-9.

2. Burt FJ, Rolph MS, Rulli NE, Mahalingam S, Heise MT Chikungunya?: a re-emerging virus. 2012;379:22-4.

3. Charrel RN, Ph D, Lamballerie X De, Ph D, Raoult D, Ph D Chikungunya Outbreaks - The Globalization. 2007;769-71.

4. Iqbal H, Aziz H. Chikungunya spurt in Pakistan: Current Status. 2017;

5. Dorji T, Tobgay T, Dorji T. Chikungunya Fever Outbreak, Bhutan, 2012. 2013;19(10):1681-4.

6. Aamir UB, Badar N, Salman M, Ahmed M, Alam MM Outbreaks of chikungunya in Pakistan. Lancet Infect Dis [Internet]. 2017;17(5):483. Available from: http://dx.doi.org/10.1016/S1473-3099(17)30191-3

7. Townson H, Nathan MB. Resurgence of chikungunya. 2008;(October 2007)

8. Ali I, Dasti JI. Chikungunya virus?; an emerging arbovirus in Pakistan. 2018;68(2):252-7.

9. Mallhi TH. Commentary?: outbreak of Chikungunya in
Pakistan. 2017;5(September):5-6.

10. Mallhi TH, Khan YH, Khan AH, Tanveer N, Qadir MI. First chikungunya outbreak in Pakistan?: a trail of viral attacks. New Microbes New Infect [Internet]. 2017;19:13-4. Available from: http://dx.doi.org/10.1016/ j.nmni.2017.05.008

11. Rauf M, Manzoor S, Mehmood A, Bhatti S. Outbreak of chikungunya in Pakistan Isoniazid-resistant tuberculosis treatment. 2017;17(March):2017.

12. Review P.clinical features of chikungunya infection in srilanka.2014;4(2):131-4

Jawed S, Khaliq S, Abid M, Shaikh JR, Salam O, Abid Z. Knowledge of Chikungunya Fever Among Medical Students of Jinnah Sindh Medical. 2018;10(8)

14. Wahid B, Ali A, Ra S, Idrees M. International Journal of Infectious Diseases Global expansion of chikungunya virus?: mapping the 64-year history. 2017;58:69-76.

15. Dinkar A, Singh J, Prakash P, Das A, Nath G. Journal of Infection and Public Health Hidden burden of chikungunya in North India?; A prospective study in a tertiary care centre. J Infect Public Health [Internet]. 2018;11(4):58691. Available from: http://dx.doi.org/10.1016/ j.jiph.2017.09.008

16. https://www.ncbi.nlm.nih.gov/pubmed/29956428 